\title{
Central autonomic nervous system response to autonomic challenges is altered in patients with a previous episode of Takotsubo cardiomyopathy
}

European Heart Journal: Acute Cardiovascular Care 2016, Vol. 5(2) 152-163

(C) The European Society of Cardiology 2015

Reprints and permissions:

sagepub.co.uk/journalsPermissions.nav

DOI: 10.1 I77/2048872615568968

acc.sagepub.com

(SAGE

\author{
Vitor H Pereira'1,2,3, Paulo Marques',2, Ricardo Magalhães ${ }^{1,2}$, \\ João Português ${ }^{3}$, Lucy Calvo ${ }^{3}$, João J Cerqueira' ${ }^{1,2}$ and Nuno Sousa ${ }^{1,2}$
}

\begin{abstract}
Aims: Takotsubo cardiomyopathy is an intriguing disease characterized by acute transient left ventricular dysfunction usually triggered by an episode of severe stress. The excessive levels of catecholamines and the overactivation of the sympathetic system are believed to be the main pathophysiologic mechanisms of Takotsubo cardiomyopathy, but it is unclear whether there is a structural or functional signature of the disease. In this sense, our aim was to characterize the central autonomic system response to autonomic challenges in patients with a previous episode of Takotsubo cardiomyopathy when compared with a control group of healthy volunteers.

Methods and results: Functional magnetic resonance imaging ( $\mathrm{FMRI}$ ) was performed in four patients with a previous

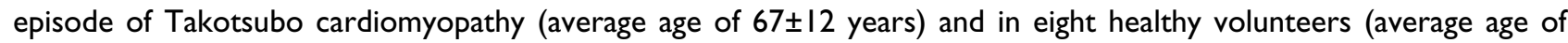
$66 \pm 5$ years) while being submitted to different autonomic challenges (cold exposure and Valsalva manoeuvre). The fMRI analysis revealed a significant variation of the blood oxygen level dependent signal triggered by the Valsalva manoeuvre in specific areas of the brain involved in the cortical control of the autonomic system and significant differences in the pattern of activation of the insular cortex, amygdala and the right hippocampus between patients with Takotsubo cardiomyopathy and controls, even though these regions did not present significant volumetric changes.

Conclusion: The central autonomic response to autonomic challenges is altered in patients with Takotsubo cardiomyopathy, thus suggesting a dysregulation of the central autonomic nervous system network. Subsequent studies are needed to unveil whether these alterations are causal or predisposing factors to Takotsubo cardiomyopathy.
\end{abstract}

\section{Keywords}

Central autonomic nervous system, sympathetic nervous system, insular cortex, Takotsubo cardiomyopathy, functional magnetic resonance imaging, stress

Date received: 29 September 20I4; accepted: 2 January 2015

\section{Introduction}

The contribution of psychological/social stress on cardiovascular diseases is currently indisputable, based on reliable evidences from basic and clinical studies. ${ }^{1,2}$ The most obvious association between stress and cardiovascular disease is the Takotsubo cardiomyopathy (TTC), also known as stress cardiomyopathy. It affects predominantly post-menopausal women with an estimated frequency of 5.2 per 100,000 patients for women and 0.6 per 100,000 patients for men in the United States. ${ }^{3}$ Clinically, TTC mimics acute myocardial
'Life and Health Sciences Research Institute (ICVS), School of Health Sciences, University of Minho, Braga, Portugal

${ }^{2}$ ICVS/3B's - PT Government Associate Laboratory, Braga/Guimarães, Portugal

${ }^{3}$ Cardiology Department, Alto Ave Hospital Centre, Guimarães, Portugal

\section{Corresponding author:}

Nuno Sousa, University of Minho, Instituto de Ciências da Vida e Saúde (ICVS), Campus de Gualtar, Universidade do Minho, 47 I0-057 Braga, Portugal.

Email: njcsousa@ecsaude.uminho.pt 


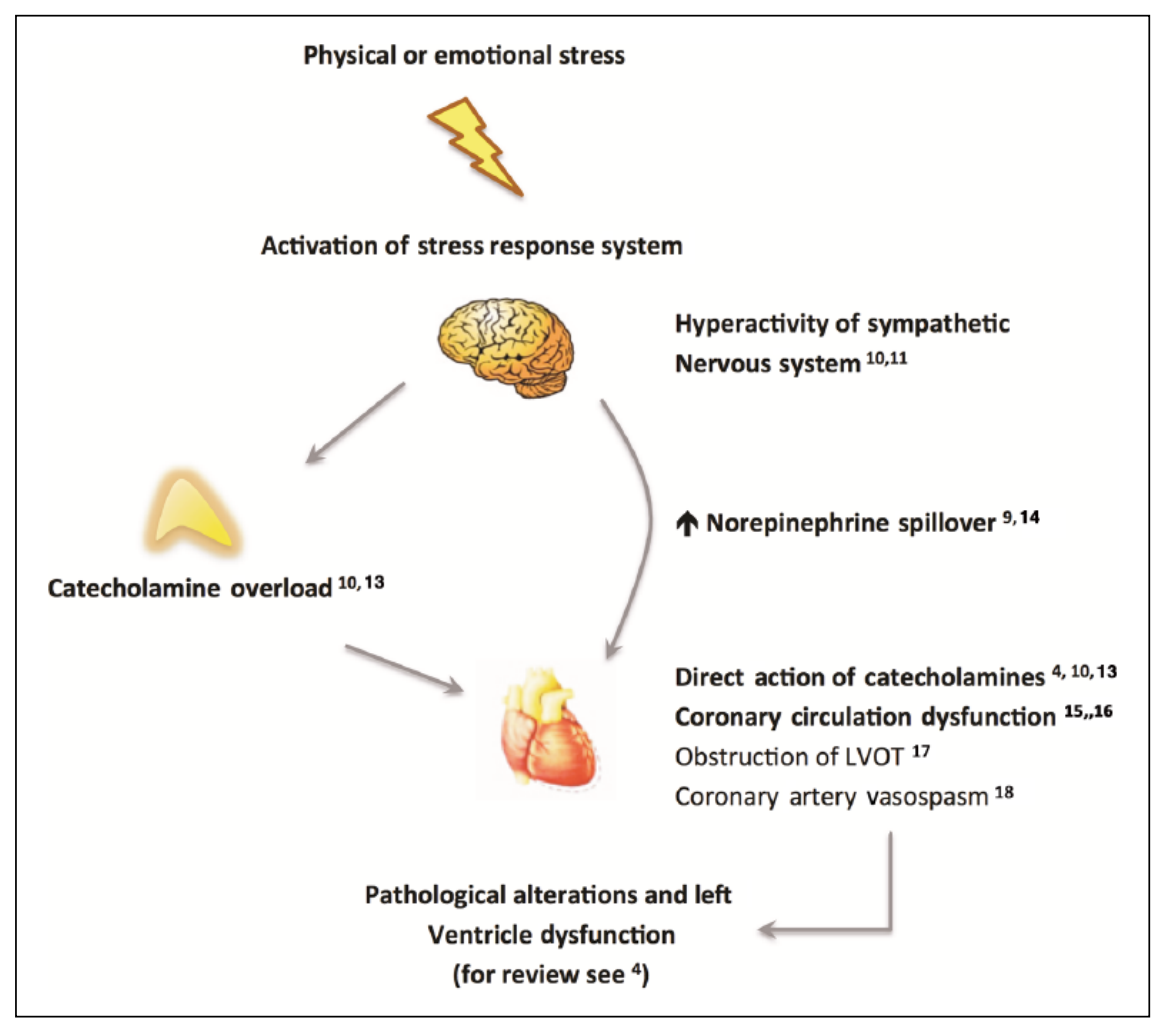

Figure I. Pathophysiologic mechanisms responsible for Takotsubo cardiomyopathy. Physical or emotional stressors are the usual triggers of this syndrome. This is followed by a hyperactivity of the sympathetic system, which, acting directly in the heart by the release of norepinephrine or through the release of catecholamines by the adrenal gland, promotes direct damage to the cardiomyocytes together with coronary circulation dysfunction, left ventricle outflow tract (LVOT) obstruction and eventually coronary vasospasm leading to left ventricle dysfunction and apical ballooning.

LVOT - Left Ventricle Outflow Tract.

infarction and is characterized by the sudden onset of chest pain accompanied by segment ST-T elevation on the ECG, mild elevation of cardiac biomarkers, transient regional left ventricle dysfunction and, importantly, absence of significant coronary artery disease on the coronariography. ${ }^{4}$ While very frequently triggered by an episode of acute emotional or physical stress, about one-third of the patients do not have a well-documented stressor and, in addition, other triggers commonly related to central nervous system related disorders (e.g. anti-depressants withdrawal or subarachnoid haemorrhage) - have been reported in the literature..$^{5-7}$ These findings support the theory that TTC may have a neurocardiogenic aetiology mediated by an overactivation of the sympathetic system that will ultimately lead to an increase in the catecholaminergic levels and induce myocardial damage. ${ }^{8}$ This theory is further reinforced by clinical studies showing that acute-phase TTC patients have an overactivation of the sympathetic system with higher levels of catecholamines when compared with patients admitted for myocardial infarction in Killip class III. ${ }^{9}$ More recently, imaging studies with positron emission tomography (PET) and single-photon emission computed tomography (CT) also suggest the presence of autonomic imbalance in the acute phase of the disease. ${ }^{10,11}$ Animal models of TTC, which use immobilization stress to induce a similar phenotype, show significant sympatho-adrenal activation comparable to that seen in human patients. ${ }^{4,12}$

In spite of these theories much is yet undetermined regarding the involvement of the autonomic control in the pathophysiology of TTC (summarized in Figure 14,9-11,13${ }^{18}$ ). In particular it is not known whether the autonomic system is altered only in the acute phase or if TTC patients have constitutional alterations in the autonomic nervous system. Favouring the latter is the fact that TTC patients are more prone to anxiety, depression and stress, suggesting they may have a state of sympathetic overactivation or increased cardiovascular reactivity. ${ }^{3}$ Interestingly, from a neuroanatomical perspective, the central areas responsible for the regulation of the autonomic system such as the insular cortex, the amygdala, the hippocampus and the cingulate cortex ${ }^{19}$ were shown to be structurally and functionally affected in anxiety related disorders, ${ }^{20}$ with a specific relevance to the insular-amygdala connectivity. ${ }^{21}$ For these reasons we hypothesize that these regions may be affected in TTC patients and thus underlie stress-related cardiovascular diseases. 
In this sense, the main goal of the current study was to characterize the central autonomic system response to autonomic challenges in specific brain regions (insular cortex, amygdala, cingulate cortex, hippocampus and prefrontal cortex) involved in the cortical regulation of the autonomic system using structural and functional magnetic resonance imaging (fMRI) in patients with a previous episode of TTC when compared with healthy controls.

\section{Material and methods}

\section{Ethics statement}

The present study was approved by the Ethics Committee of Hospital de Braga and Hospital of Guimarães (Portugal) and was conducted in accordance with the principles expressed in the Declaration of Helsinki. The study goals and tests were explained to all participants, who all gave informed written consent.

\section{Subjects}

Ten patients with a previous episode of TTC and 10 sexand age-matched healthy controls were recruited for the study. The subjects were all post-menopausal women with a well-documented episode of TTC triggered by an emotional stressor. From the initial group of 10 TTC patients only four were enrolled in the study as three patients had absolute contra-indications for the realization of fMRI; one was lost for follow-up and two refused to participate. The remaining four patients included had normal left ventricular function and were under no beta-blockers. The mean age of the control group was $66 \pm 5$ years and the mean age of the TTC group was $67 \pm 12$ years $(p=0.77)$. All individuals in the control group were recruited from the general population of the region of Braga, Portugal. The volunteers had no relevant past medical history including stroke, myocardial infarction or heart failure. While the patients had not been submitted to a psychological analysis previous to the fMRI, none of them had a clinical diagnosis of depression or anxiety-related disorders. Social status was similar in all participants.

\section{MRI acquisition protocol}

All subjects underwent the same MRI acquisition protocol and were screened on a clinical approved Magnetom Avanto 1.5T (Siemens Medical Solutions, Erlangen, Germany) scanner at Hospital de Braga using a Siemens 12-channel receive-only head coil. Foam pads were used in order to minimize head movement during acquisition. A 3D T1-weighted magnetization prepared rapid gradient echo (MPRAGE) structural scan was acquired with the following parameters: repetition time $(T R)=2.730 \mathrm{~s}$, echo time $(T E)=3.48 \mathrm{~ms}$, flip angle $=7^{\circ}, 176$ sagittal slices, in-plane resolution $=1.0 \mathrm{~mm} \times 1.0 \mathrm{~mm}$ and slice thickness $=1.0 \mathrm{~mm}$. Additionally, two T2* weighted gradient-echo echo planar imaging (EPI) functional scans were acquired with the same parameters: 172 volumes, $T R=2.5 \mathrm{~s}, T E=$ $30 \mathrm{~ms}$, flip angle $=90^{\circ}$, in-plane resolution $=3.0 \times 3.0$ $\mathrm{mm}^{2}$, imaging matrix $=64 \times 64$, field of view $($ Fov $)=192$ $\mathrm{mm} \times 192 \mathrm{~mm}$, slice thickness $=4 \mathrm{~mm}$ and 38 interleaved axial slices.

During the first functional acquisition subjects were presented with a visual cue that instructed them to rest during a baseline period of $60 \mathrm{~s}$. After this period, another visual cue was presented indicating that they should start performing the Valsalva manoeuvre and the cue was maintained for a period of $17.5 \mathrm{~s}$. This procedure was repeated four times and was followed by a $120 \mathrm{~s}$ additional baseline period in order to improve the baseline estimate. In the second functional scan, a similar procedure to the one described above was employed, but the Valsalva manoeuvre was replaced by a stressful stimulus. In the present study ice blocks placed at the sole of the right foot were used as stressors. The visual cues were presented using the fully integrated and synchronized fMRI system IFIS-SA.

\section{Heart rate and Valsalva manoeuvre}

Heart rate was measured throughout the scan using a MRIcompatible pulse oximeter in the right index finger and the heart rate calculated using a peak-detection algorithm. Subjects practised the Valsalva manoeuvre outside the scanner for procedure familiarization, as described elsewhere. ${ }^{22}$ A patient from each group did not perform the Valsalva manoeuvre correctly and was excluded from the fMRI analysis during this task. Another patient from the control group was excluded due to diffuse cerebrovascular disease and severe cortical atrophy, what gives a total of eight controls and three TTC patients analysed during the Valsalva manoeuvre.

\section{Data pre-processing}

Before any data processing and analysis, all acquisitions were visually inspected by a certified neuroradiologist and it was confirmed that they were not affected by critical head motion and that participants had no brain lesions.

The functional datasets were pre-processed using FMRIB Software Library (FSL) version 5.0.4. Preprocessing steps included motion correction, slice timing correction, skull stripping, and spatial smoothing with a 8 mm Full-Width at Half-Maximum (FWHM) Gaussian kernel. Additionally, global scaling, a detrending procedure, was applied by dividing each voxel of each brain volume by the mean of the corresponding brain volume. No subject exhibited head motion higher than $2 \mathrm{~mm}$ in translation or $1^{\circ}$ in rotation. 


\section{Region of interest analysis}

Region of interest (ROI) analyses were performed in order to inspect the time course responses at several brain regions. Firstly, the structural scans were segmented using the semiautomated workflow implemented in Freesurfer toolkit version 5.1 (http://surfer.nmr.mgh.harvard.edu). The workflow and procedures employed were validated against manual segmentation procedure ${ }^{23}$ and are considered reliable across sessions, scanner platforms, updates and field strengths. ${ }^{24,25}$ For the present study the segmentation according to the Desikan atlas was considered. The segmented structural images were then co-registered to the functional scans. Then, masks for the left and right insular, amygdala, hippocampus, ventromedial prefrontal cortex (defined as the sum of the lateral and medial orbitofrontal cortex) and several sub-regions of the cingulate cortex (i.e. rostral-anterior, caudal-anterior, isthmus and posterior divisions) were created by thresholding the structural segmented image at the intensities of the corresponding structure. The average time series of each ROI was then extracted using the fslmeants tool distributed with FSL, which extracts the average time series over all voxels in the corresponding mask.

Freesurfer segmentation procedures also resulted in the estimation of subcortical, white matter and cortical ROI volumes. Volumes from the above mentioned regions were considered for ROI-wise volumetric analysis.

\section{Statistical analysis}

The numeric and statistical analysis was performed in Matlab R2009a and Prism6. First, for each subject, each time series was converted to percentage of signal change relative to the baseline periods and the four Valsalva/cold exposure periods were averaged. Mann-Whitney $U$ tests were performed at each time-point in order to inspect whether the blood oxygen level dependent (BOLD) response to the Valsalva manoeuvre differed between the control group and the experimental group. This procedure was performed for time series with and without global scaling. A similar procedure was applied in order to investigate whether the heart rate of the participants differed from the baseline rates during the Valsalva/ stress periods. Additionally, mixed-design Analyses of Variance (ANOVAs) were performed in order to test for differences in each region between left and right hemispheres and for group by hemisphere interactions. For the volumetric analysis, each ROI volume was adjusted for head size by dividing each regional volume by the intracranial volume (ICV), thus reflecting percentages of ICV. Then, MannWhitney $U$ tests were performed using the ICV corrected regional volumes in order to investigate possible volumetric differences between groups. All the cases are plotted in the respective graphs and the line represents the median. $p$ value was considered a trend when less than 0.1 and significant when less than 0.05 . The size effects were calculated using common language effect size and are reported along the text when the $p$ value is significant. Corrections for multiple comparisons were not performed due to the small sample size.

\section{Results}

\section{Volumetry}

No differences were found in the volumes of the ROIs studied (described above) when normalized to the total ICV with the exception of the left amygdala, which had a lower volume in TTC patients when compared with controls (control: $0.1016 \pm 0.004 \%$, TTC: $0.076 \pm 0.003 \%$; $p=0,015$; Figure 2).

\section{Heart rate response to Valsalva manoeuvre and cold exposure}

The Valsalva manoeuvre elicited an expressive variation in the heart rate relative to the baseline in both groups (Figure 3(a)). In the control group the heart rate significantly increased after the beginning of the of the Valsalva manoeuvre until the end of the strain phase, when it dropped slightly, rising again until it peaked at $22.5 \mathrm{~s}$ ( $\%$ change: $15.8 \% ; p<0,05$; relative to baseline). This peak was followed by a reflex bradycardia that extended until $40 \mathrm{~s}$, after which the heart rate returned to basal levels. This pattern of response was similar in the TTC group; however, for these patients, the peak after the strain was reached later $(25 \mathrm{~s} ; \%$ change: $17.1 ; p<0,05$; relative to baseline) and there was no reflex bradycardia (Figure 3(a)).

During cold exposure there were slight variations of the heart rate, albeit not statistically significant when compared with the basal heart rate previously recorded before the exposure (Figure 3(b)).

\section{BOLD signal response to the Valsalva manoeuvre}

The changes elicited by the Valsalva manoeuvre displayed a similar pattern in all brain regions studied (Figure 4(a) and (b); data from the insular cortex are representative of what was observed for other regions). Briefly, there was an increase in the BOLD signal after the beginning of the manoeuvre followed by an undershoot that persisted until the release of the strain when the BOLD signal raised again, peaking at $25 \mathrm{~s}$ and returning to normal 30-35 s after the start of the task. These variations in the BOLD signal most probably reflected the changes in the global perfusion of the brain provoked by blood pressure fluctuations induced by the Valsalva manoeuvre. To correct for this confounding factor the BOLD signal of each ROI was normalized to the average signal of the whole brain. 


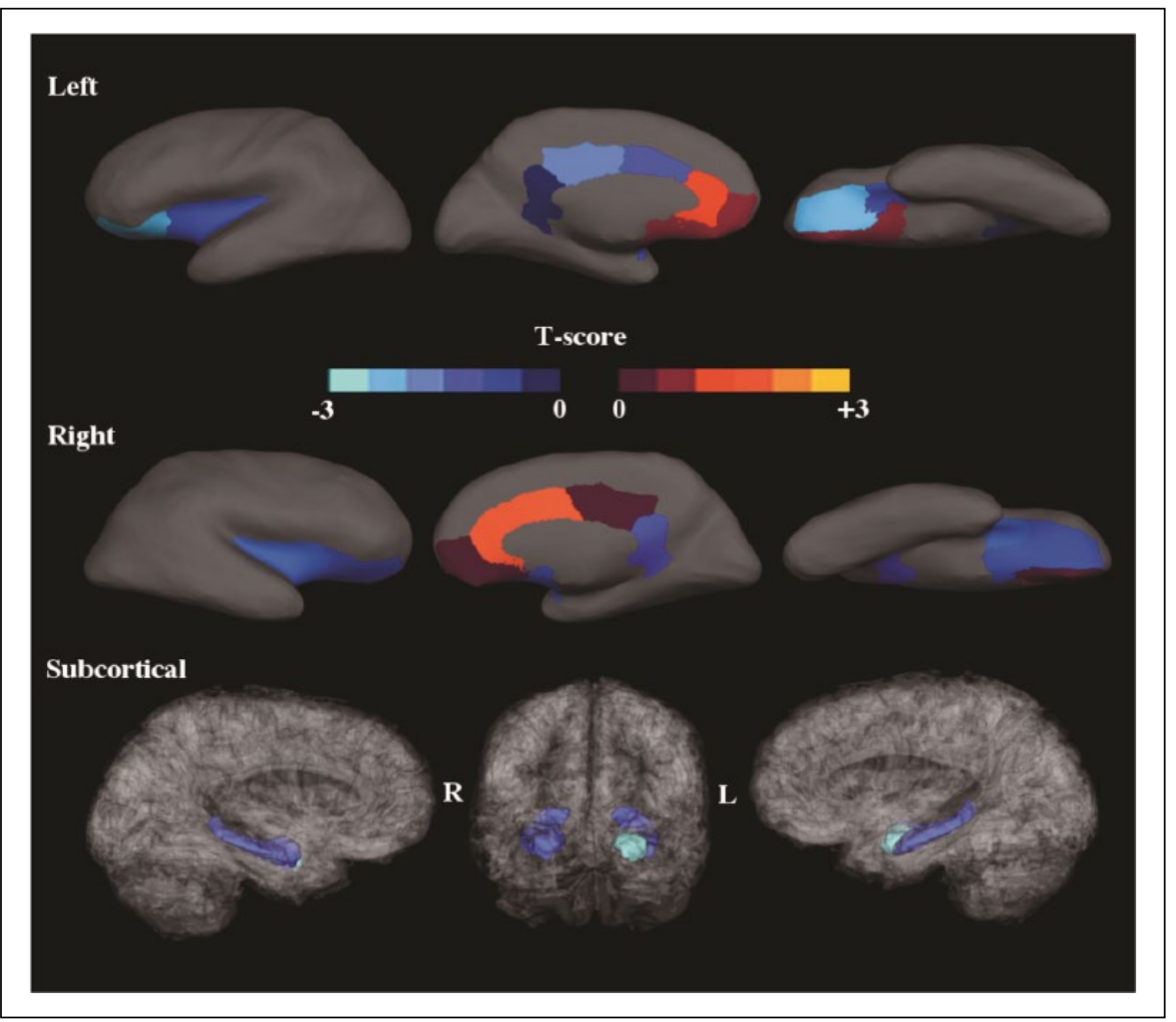

Figure 2. Volumetric changes in the brain between controls and Takotsubo patients. All the areas analysed are represented in the images and were coloured according with the $T$-value (blue means that the volume was higher in controls and yellow means that the volume was lower in controls). No differences were found in the volumes when normalized to the intracranial volume (ICV) with the exception of the left amygdala (light blue), which had a lower volume in Takotsubo patients when compared with controls (control: $0.1016 \pm 0.004 \%$ of ICV; Takotsubo: $0.076 \pm 0.003 \%$ of ICV; $p=0.004$ ).

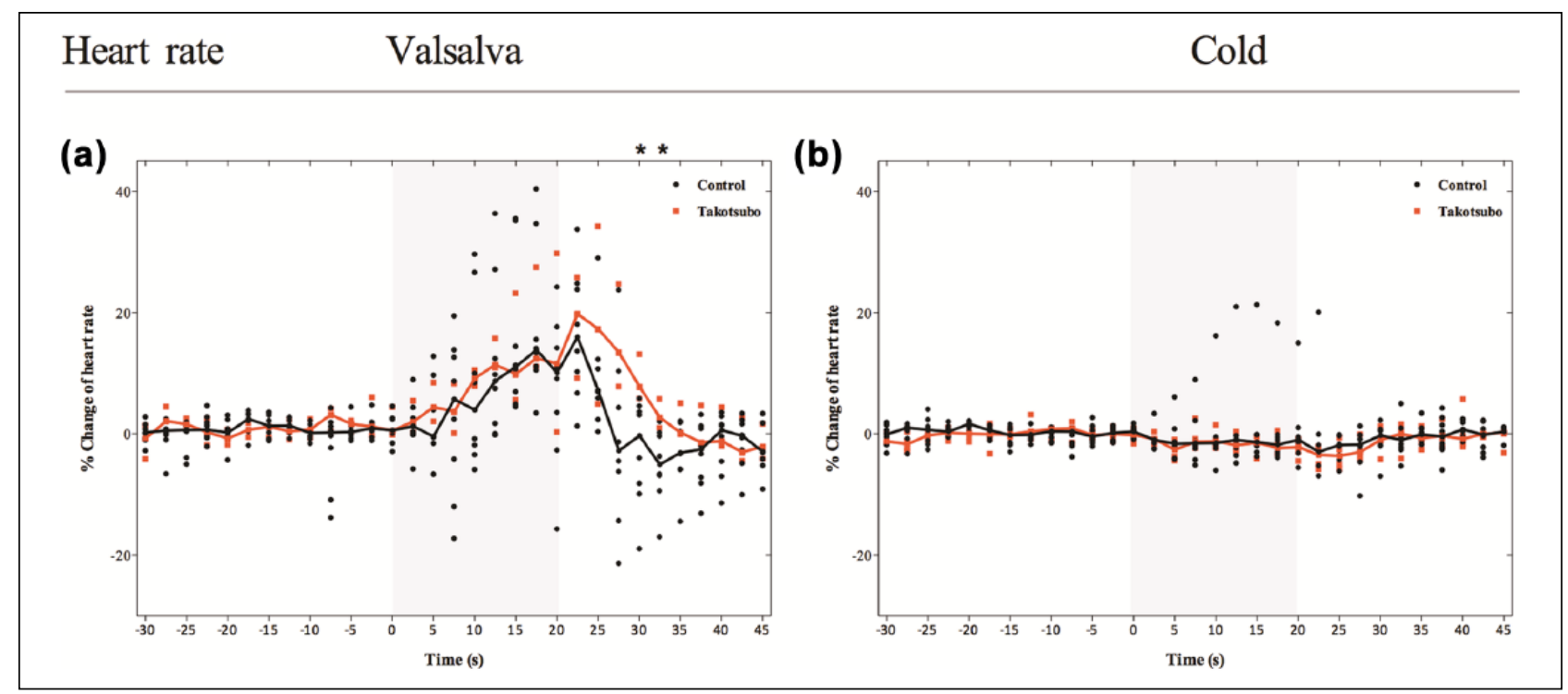

Figure 3. Variation of heart rate (expressed in percentage change towards baseline) during the Valsalva manoeuvre and during cold exposure. During the Valsalva manoeuvre the heart rate increases during the strain phase peaking $22.5 \mathrm{~s}$ after the beginning of the manoeuvre. This peak is followed by a rapid decline followed by a reflex bradycardia (a). In Takotsubo cardiomyopathy (TTC) patients the peak is reached later and there is no reflex bradycardia (a). During cold exposure no significant changes were elicited in the heart rate in both controls and TTC patients (b). Asterisks represent significant differences between the groups $(p<0.05)$. 


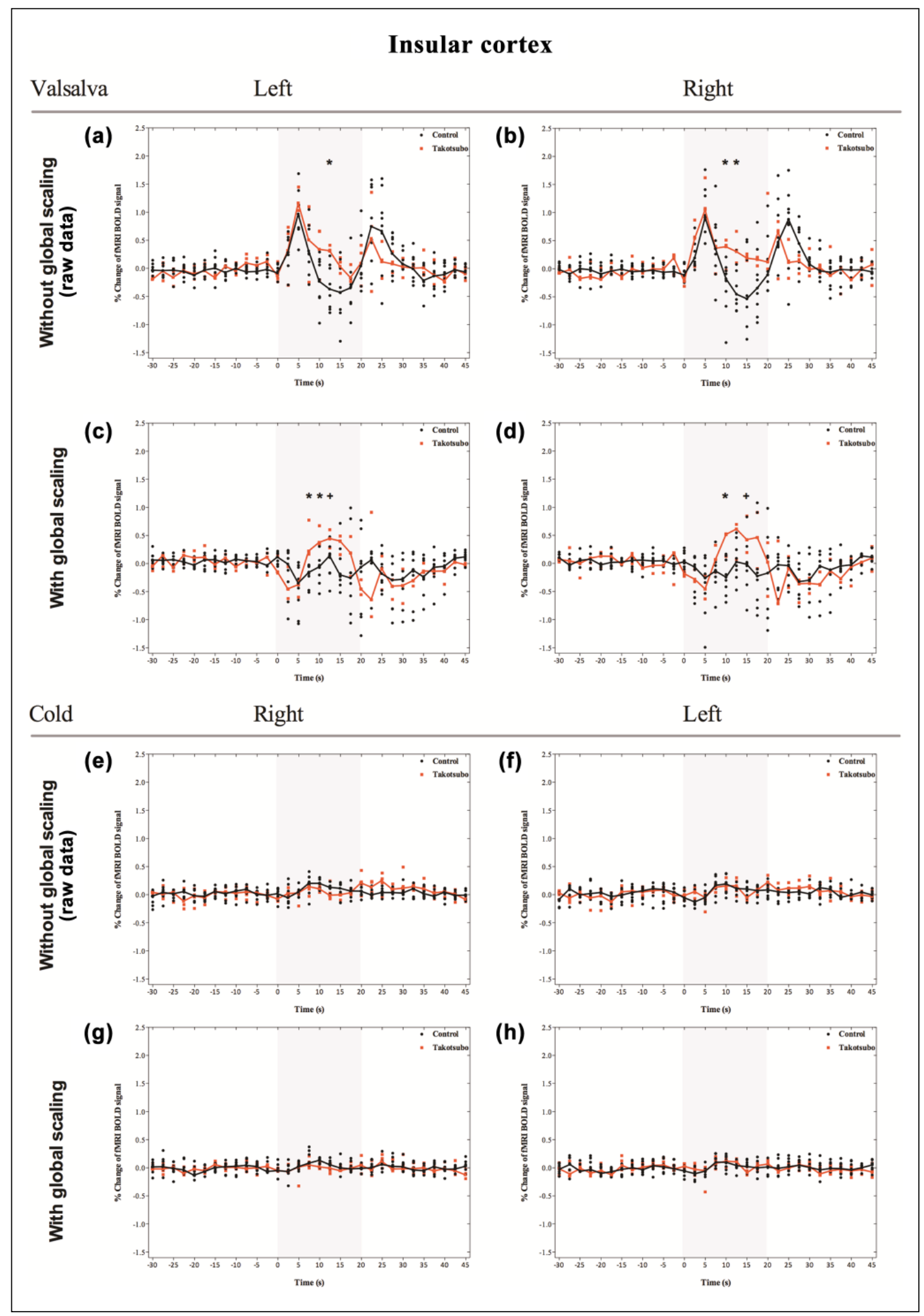

Figure 4. Blood oxygen level dependent (BOLD) signal changes relative to baseline during Valsalva manoeuvre and cold exposure in the right and left insular cortex. During the Valsalva manoeuvre there is an increase in the BOLD signal followed by an undershoot until the release of the strain when another positive peak is recorded before the return to baseline. This response is similar on the left (a) and on the right (b). After global scaling a change in the response magnitude is noted during the strain phase of the Valsalva manoeuvre in TTC patients on both hemispheres ((c) and (d)). During cold exposure there were no significant variation of the BOLD signal neither between groups nor when compared with baseline $((\mathrm{e})$ to $(\mathrm{h}))$. Asterisks mark the time points were both the $p$-value was less than 0.05 and the $r$-value (effect size) was superior to 0.8 . 


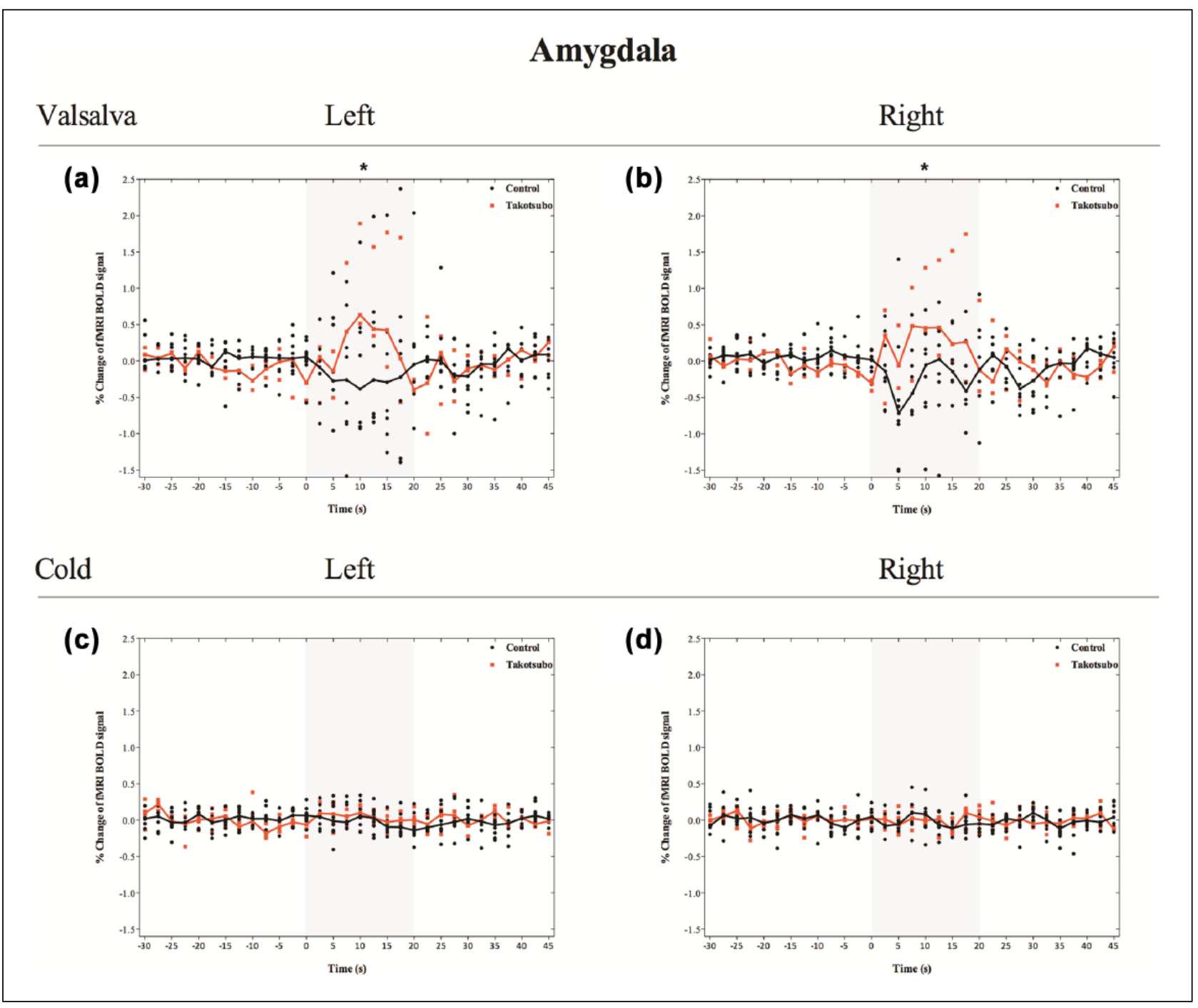

Figure 5. Blood oxygen level dependent (BOLD) signal changes relative to baseline during Valsalva manoeuvre and cold exposure in the right and left amygdala after global scaling. There was a difference in the pattern of response to the Valsalva manoeuvre between the groups in the amygdala bilaterally ((a) and (b)). While in TTC there was a positive change in the BOLD signal, in the control groups the Valsalva manoeuvre elicited an overall negative response. During cold exposure there was no significant variation of the BOLD signal neither between groups nor when compared with baseline ((c) and (d)). Asterisks mark the time points where both the $p$-value was less than 0.05 and the $r$-value (effect size) was superior to 0.8 .

Following the normalization (global scaling) of each ROI to the whole brain signal we analysed the BOLD signal response to the Valsalva manoeuvre in specific brain regions. In the insular cortex there was a significant difference in the magnitude of the response between control and TTC groups. After the beginning of the manoeuvre there was a decrease in the BOLD signal in both groups followed by an increase in the signal, which was significantly more pronounced in the TTC group. As we can see in Figure 3(c) and (d) these differences were more prominent on the left side at $7.5 \mathrm{~s} \mathrm{( \%} \mathrm{of} \mathrm{change:} \mathrm{control,} 0.165 \%$; TTC, $0.215 \% ; p<0.05 ; r=1$ ), $10 \mathrm{~s}$ (\% of change: control, $-0.07 \%$; TTC, $0.38 ; p<0.05 ; r=1)$ and $12.5 \mathrm{~s}(\%$ of change: control, $-0,131 \%$; TTC, $0.44 \% ; p<0.1 ; r=0.85$ ); and on the right side at $0 \mathrm{~s}$ ( $\%$ of change: control, $0.03 \%$; TTC,
$-0.18 \% ; p<0.05 ; r=0.041), 10 \mathrm{~s}$ ( $\%$ of change: control, $-0.25 \%$; TTC, $0.55 \% ; p<0.05 ; r=0.91)$ and $15 \mathrm{~s}(\%$ of change: control: $-0.02 \%$; TTC, $0.42 \% ; p<0.1 ; r=0.875$ ). After the strain release the BOLD signal resumed to the same levels as the control group until complete normalization to baseline levels.

The pattern of response of the amygdala to the Valsalva manoeuvre was also different in both groups although it missed being statistically significant (Figure 5(a) and (b)). While in control groups the BOLD signal displayed a negative response, which was more pronounced after the end of the manoeuvre, in the TTC group the Valsalva manoeuvre elicited a positive variation of the BOLD signal bilaterally. The differences between the groups were more evident at $10 \mathrm{~s}$ after the beginning of the task bilaterally (\% of change: 
left - control, $-0.39 \%$; TTC, $0.63 \% ; p<0.05 ; r=0.92$; right - control, $-0.06 \%$; TTC, $0.45 \% ; p<0.05 ; r=0.92$ ) (Figure 5 (a) and (b)).

The cingulate cortex was divided into four regions: the anterior cingulate cortex, the caudal anterior cingulate cortex, the posterior cingulate cortex and the isthmus of the cingulate cortex. The former displayed an apparent change in the magnitude of the response of the BOLD signal not only during the Valsalva manoeuvre but also after its end (Figure 6(a) to (d)). In spite of this observation this difference was not statistically significant.

The ventral medial prefrontal cortex and the hippocampus also contribute to the central autonomic nervous system. While the former displayed no significant variations of the BOLD signal relative to baseline (data not shown), in the hippocampus there were changes in the magnitude and timing of response in the TTC group. These differences were exclusive of the right side and were more prominent at $10 \mathrm{~s}$ ( $\%$ of change: control, $-0.29 \%$; TTC, $0.32 \% ; p<0,05$; $r=1$ ) (Figure 7(a) and (b)).

\section{BOLD signal response to cold exposure}

Cold exposure elicited a positive increase in the BOLD signal after 7.5 and $10 \mathrm{~s}$ in the left and right insular cortex in both groups (Figure 4(e) to (h)). In the other regions cold exposure did not elicit any significant response on both sides. This observation remained after normalization of the data for the average of the whole brain signal (Figure 4(e) to $(\mathrm{h})$; data from the insular cortex is representative of what was observed for other regions). There were no significant variations of the BOLD signal between control and TTC groups in any of the regions studied.

\section{Discussion}

The central autonomic nervous system is organized in a complex and intricate network formed by cortical and subcortical regions such as the insular cortex, anterior and midcingulate cortices, amygdala, ventral medial prefrontal cortex, hippocampus formation and the hypothalamus. ${ }^{26}$ Through their projections with limbic structures and medullary centres responsible for the afferent and efferent pathways of the peripheral nervous system, these regions are able to integrate emotional and superior cerebral functions like decision-making or cognition in the autonomic response. ${ }^{27}$ Herein, we showed that some of these areas specifically the insular cortex, the amygdala and the right hippocampus - respond abnormally to the Valsalva manoeuvre in patients with a previous episode of TTC. These findings gain greater relevance in light of the growing body of evidence showing that the sympathetic system is implicated in the pathophysiology of this disease. In line with this perspective, Vaccaro and colleagues showed that acute-phase patients admitted for TTC had elevated sympathetic activity associated with a decrease in spontaneous baroreflex control of sympathetic activity. ${ }^{10}$ Interestingly, the insular cortex (the region where the differences between controls and TTC patients were more prominent in our study) has been recognized to play a critical role in baroreflex control due to the presence of both sympathoexcitatory and sympathoinhibitory neurons responsive to baroreceptor stimulation in both rodents and monkeys. ${ }^{28,29}$ Human studies have largely validated these findings showing that this region is involved in the afferent and efferent pathways of the autonomic system, implying the insular cortex as the main regulator of the cortical autonomic response (for review see Palma and Benarroch $^{27}$ and Nagai et al. ${ }^{30}$ ). Accordingly, patients with acute stroke with extension to the insular cortex are more prone to sudden cardiac death and cardiac arrhythmias ${ }^{31,32}$ and a case report of TTC following cerebral infarction involving the insular cortex has been published in the literature. ${ }^{33}$ fMRI studies have also demonstrated that the insular cortex is recruited in response to autonomic challenges, and a gyri-specific functional organization of this region to specific autonomic challenges has been proposed. ${ }^{34}$ Although we did not segment the insular cortex into its different gyri, which may make difficult the interpretation of our data, we showed a change in the magnitude response of both insular cortices during the strain phase of the Valsalva manoeuvre in TTC patients. This was paralleled by a time-different heart rate response to the Valsalva manoeuvre in the TTC group; in fact, TTC patients had a more sustained increase in heart rate and did not display the typical reflex bradycardia usually observed in phase IV of this manoeuvre, a response that is dependent on the parasympathetic system. However, we did not thoroughly evaluate the autonomic function in our subjects and heart rate was the only outcome studied precluding a definite conclusion regarding the real value and significance of this alteration in the heart rate response, which is a limitation of our study. In addition, we did not find any statistically significant differences in the BOLD signal change during phase IV of the Valsalva manoeuvre in any of the regions studied. Further studies using specific tools to measure the autonomic response during the fMRI may be valuable to clarify this topic.

The insular cortex has important interconnections with the amygdala, the anterior cingulate cortex and the hippocampus. These regions have been proved to be involved in the regulation of cardiac control in several imaging studies. ${ }^{26}$ Accordingly, we also showed a change in the pattern of the response of the amygdala bilaterally and of the right hippocampus during the strain phase of the Valsalva. It is interesting to note that these regions have been ascribed with important roles in the emotional response and that our patients had previously had an episode of TTC triggered by an emotional stressor. We did not find any differences in the BOLD signal response to the Valsalva manoeuvre in other regions, suggesting that TTC patients may have a specific disruption of the insular-amygdalar and hippocampal 


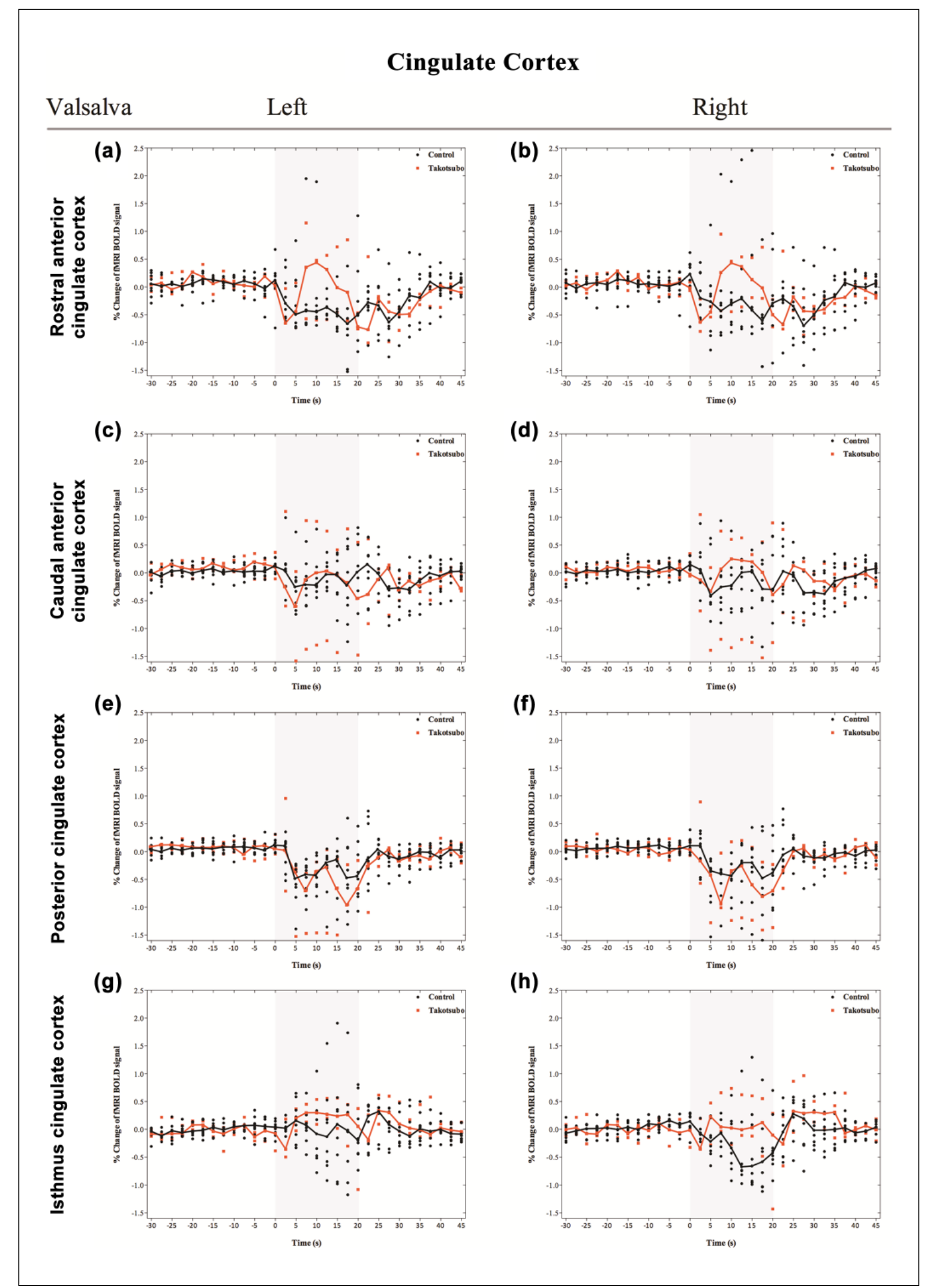

Figure 6. Blood oxygen level dependent (BOLD) signal changes relative to baseline during Valsalva manoeuvre in the different regions of the cingulate cortex. No significant differences occurred between groups in response to Valsalva manoeuvre in the rostral anterior cingulate cortex ((a) and (b)), caudal anterior cingulate cortex ((c) and (d)), isthmus cingulate cortex $((e)$ and (f)) and posterior cingulate cortex $((\mathrm{g})$ and $(\mathrm{h}))$. Asterisks mark the time points where both the $p$ value was less than 0.05 and the $r$ value (effect size) was superior to 0.8 . 


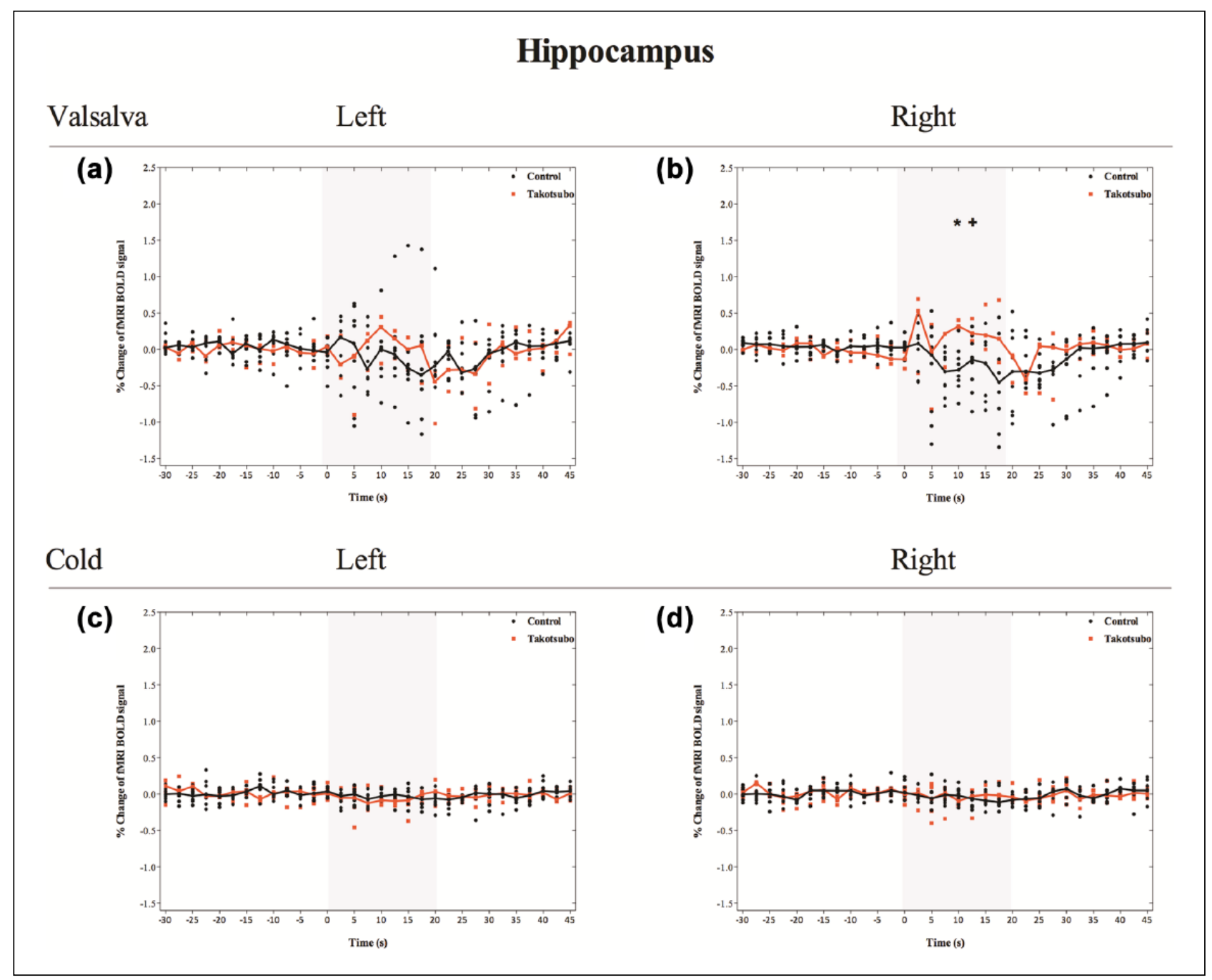

Figure 7. Blood oxygen level dependent (BOLD) signal changes relative to baseline during Valsalva manoeuvre and cold exposure in the right and left hippocampus after global scaling. There was a difference in the magnitude of the response to the Valsalva manoeuvre between the groups in the right hippocampus (b), but not in the left hippocampus (a). While in the control groups the Valsalva manoeuvre did not elicited a significant change in BOLD signal, in TTC there was a positive change in the BOLD signal in the right hippocampus (b). During cold exposure there were no significant variations of the BOLD signal neither between groups nor when compared with baseline ((c) and (d)). Asterisks mark the time points were both the $p$ value was less than 0.05 and the $r$-value (effect size) was superior to 0.8 .

connection. Interestingly an fMRI study in women with posttraumatic stress disorder also showed an exaggerated insular-amygdalar BOLD response to stressful cues thus suggesting that these regions may underlie stress-related diseases. ${ }^{35}$ Similarly, fMRI studies, using paradigms of mental stress, attributed the autonomic alterations recorded during those tasks to an activation of the right insular cortex, ${ }^{36}$ which also suggests that this region may be an important link between stress and cardiovascular disease. From a different perspective we should also consider the fact that these alterations in the BOLD signal might be related to differences in the anxious state between controls and TTC patients since some studies show that even an anxious trait are sufficient to induce functional and structural alterations in several brain regions and more specifically in the amygdala and the insular cortex. ${ }^{37-39}$ While our patients did not present clinical signs of anxiety, this point should be addressed in future studies.

During cold exposure there was no significant variation in the BOLD signal in any region evaluated, which differs from results from a previous study. ${ }^{34}$ However, this difference may be justified by the short time of exposure in our study ( $20 \mathrm{~s} v s .120 \mathrm{~s})$ and due to the intensity of the stressor (in fact, many of our patients reported that the cold sensation was only mild).

This study was mainly limited by the small sample size (control $n=8$; TTC $n=3 / 4$ (Valsalva and cold respectively)), which was justified by the low incidence of 
TTC clearly triggered by emotional stressors in our centre. This was further aggravated by the refusal of some patients to perform fMRI. Even though the small sample size resulted in low power of the tests, it was enough to reveal some significant changes in areas of relevance to the topic. Corrections for multiple comparisons were not performed due to small sample size (which results in low power) and to the fact that this was an exploratory analysis over several brain regions and a wide range of timepoints (0-45 s after the beginning of the manoeuvre), which would have resulted in a high rate of Type II errors. Another limitation of this study was the fact that a psychological analysis was not performed in our participants, namely comprehensive posttraumatic stress or anxiety evaluations. This is important since these disorders may influence the activity of the same brain regions analysed in our study such as the amygdala, insular cortex and hippocampus. However, the alterations reported herein occurred specifically during the Valsalva manoeuvre, a well-recognized autonomic challenge, and are probably related with the regulation of the autonomic nervous system.

In spite of these limitations we show for the first time that in patients with TTC the central autonomic nervous system response to the Valsalva manoeuvre is altered several years after the acute event. Such dysregulation of the neural network involved in central autonomic control is likely to be critical to TTC, even though at this point one cannot determine whether it is simply a causal consequence of a previous event or whether it may constitute a predisposing factor. A longitudinal study will be needed to answer this question; similarly, to overcome the small sample of this study, a multi-centric study should be designed to enrol more patients in order to provide definite answers on the relevance of dysregulation of the central autonomous neural network in patients with TTC.

\section{Acknowledgements}

The authors are thankful to all study participants and to Dr Nadine Santos in the development of the current study. VHP and PM contributed equally to this work.

\section{Conflict of interest}

The authors declare that there is no conflict of interest.

\section{Funding}

The work was supported by the European Commission (FP7): 'SwitchBox' (Contract HEALTH-F2-2010-259772) and 'MyHealth' project (Contract DoIT-13853), and by Fundação para a Ciência e Tecnologia (project FCT-ANR/NEU-OSD/0258/2012 Portuguese North Regional Operational Program (ON.2 - O Novo Norte)) under the National Strategic Reference Framework (QREN), through the European Regional Development Fund (FEDER). PM is supported by a 'SwitchBox' fellowship and RM by a FCT-ANR/NEU-OSD/0258/2012 fellowship.

\section{References}

1. Steptoe A and Kivimäki M. Stress and cardiovascular disease. Nat Rev Cardiol 2012; 9: 360-370.

2. Pereira VH, Cerqueira JJ, Palha JA, et al. Stressed brain, diseased heart: A review on the pathophysiologic mechanisms of neurocardiology. Int J Cardiol 2013; 166: 30-37.

3. Deshmukh A, Kumar G, Pant S, et al. Prevalence of Takotsubo cardiomyopathy in the United States. Am Heart $J$ 2012; 164: 66-71.e1.

4. Nef HM, Möllmann H, Akashi YJ, et al. Mechanisms of stress (Takotsubo) cardiomyopathy. Nat Rev Cardiol 2010; 7: 187-193.

5. Porto I, Della Bona R, Leo A, et al. Stress cardiomyopathy (tako-tsubo) triggered by nervous system diseases: A systematic review of the reported cases. Int J Cardiol 2013; 167: 2441-2448.

6. Dias A, Franco E, Figueredo VM, et al. Occurrence of Takotsubo cardiomyopathy and use of antidepressants. Int $J$ Cardiol 2014; 174: 433-436.

7. Das M, Gonsalves S, Saha A, et al. Acute subarachnoid haemorrhage as a precipitant for takotsubo cardiomyopathy: A case report and discussion. Int J Cardiol 2009; 132: 283 285.

8. Pelliccia F, Greco C, Vitale C, et al. Takotsubo Syndrome (stress cardiomyopathy): An intriguing clinical condition in search of its identity. Am J Med 2014; 127: 699-704.

9. Wittstein IS, Thiemann DR, Lima JAC, et al. Neurohumoral features of myocardial stunning due to sudden emotional stress. N Engl J Med 2005; 352: 539-548.

10. Vaccaro A, Despas F, Delmas C, et al. Direct evidences for sympathetic hyperactivity and baroreflex impairment in Tako Tsubo cardiopathy. PLoS One 2014; 9: e93278.

11. Suzuki H, Matsumoto Y, Kaneta T, et al. Evidence for brain activation in patients with takotsubo cardiomyopathy. Circ $J$ 2013; 78: 256-258.

12. Ueyama T. Emotional stress-induced Tako-tsubo cardiomyopathy: Animal model and molecular mechanism. Ann NY Acad Sci 2004; 1018: 437-444.

13. Akashi YJ1, Nakazawa K, Sakakibara M, et al. The clinical features of takotsubo cardiomyopathy. QJM 2003; 96: 563-573.

14. Kume T1, Kawamoto T, Okura H, et al. Local release of catecholamines from the hearts of patients with tako-tsubo-like left ventricular dysfunction. Circ J 2008; 72: 106-108.

15. Kurisu $\mathrm{S}$, Inoue I, Kawagoe $\mathrm{T}$, et al. Myocardial perfusion and fatty acid metabolism in patients with tako-tsubo-like left ventricular dysfunction. J Am Coll Cardiol 2003; 41: $743-748$.

16. Kume $\mathrm{T}$, Akasaka $\mathrm{T}$, Kawamoto $\mathrm{T}$, et al. Relationship between coronary flow reserve and recovery of regional left ventricular function in patients with tako-tsubo-like transient left ventricular dysfunction. J Cardiol 2004; 43: 123-129.

17. El Mahmoud R, Mansencal N, Pilliére R, et al. Prevalence and characteristics of left ventricular outflow tract obstruction in Tako-Tsubo syndrome. Am Heart J 2008; 156: 543548.

18. Dote K, Sato H, Tateishi H, et al. Myocardial stunning due to simultaneous multivessel coronary spasms: A review of 5 cases. J Cardiol 1991; 21: 203-214. 
19. Cechetto DF. Cortical control of the autonomic nervous system. Exp Physiol 2014; 99: 326-331.

20. Brühl AB, Hänggi J, Baur V, et al. Increased cortical thickness in a frontoparietal network in social anxiety disorder. Hum Brain Mapp 2014; 35: 2966-2977.

21. Baur V, Hänggi J, Langer N, et al. Resting-state functional and structural connectivity within an insula-amygdala route specifically index state and trait anxiety. Biol Psychiatry 2013; 73: 85-92.

22. Ogren JA, Macey PM, Kumar R, et al. Impaired cerebellar and limbic responses to the valsalva maneuver in heart failure. Cerebellum 2012; 11: 931-938.

23. Fischl B, Salat DH, Busa E, et al. Whole brain segmentation: Automated labeling of neuroanatomical structures in the human brain. Neuron 2002; 33: 341-355.

24. Han $X$ and Fischl B. Atlas renormalization for improved brain MR image segmentation across scanner platforms. IEEE Trans Med Imaging 2007; 26: 479486.

25. Jovicich J, Czanner S, Han X, et al. MRI-derived measurements of human subcortical, ventricular and intracranial brain volumes: Reliability effects of scan sessions, acquisition sequences, data analyses, scanner upgrade, scanner vendors and field strengths. Neuroimage 2009; 46: 177192.

26. Beissner F, Meissner K, Bär K-J, et al. The autonomic brain: An activation likelihood estimation meta-analysis for central processing of autonomic function. $J$ Neurosci 2013; 33 : 10503-10511.

27. Palma J-A and Benarroch EE. Neural control of the heart: Recent concepts and clinical correlations. Neurology 2014; 83: 261-271.

28. Zhang ZH, Rashba S and Oppenheimer SM. Insular cortex lesions alter baroreceptor sensitivity in the urethane-anesthetized rat. Brain Res 1998; 813: 73-81.
29. Zhang ZH, Dougherty PM and Oppenheimer SM. Characterization of baroreceptor-related neurons in the monkey insular cortex. Brain Res 1998; 796: 303-306.

30. Nagai M, Hoshide $\mathrm{S}$ and Kario K. The insular cortex and cardiovascular system: A new insight into the brain-heart axis. $J$ Am Soc Hypertens 2010; 4: 174-182.

31. Christensen H, Boysen G, Christensen AF, et al. Insular lesions, ECG abnormalities, and outcome in acute stroke. $J$ Neurol Neurosurg Psychiatry 2005; 76: 269-271.

32. Tokgozoglu SL, Batur MK, Topcuoglu MA, et al. Effects of stroke localization on cardiac autonomic balance and sudden death. Stroke 1999; 30: 1307.

33. Cho H-J, Kim HY, Han SH, et al. Takotsubo cardiomyopathy following cerebral infarction involving the insular cortex. $J$ Clin Neurol 2010; 6: 152-155.

34. Macey PM, Wu P, Kumar R, et al. Differential responses of the insular cortex gyri to autonomic challenges. Auton Neurosci 2012; 168: 72-81.

35. Fonzo GA, Simmons AN, Thorp SR, et al. Exaggerated and disconnected insular-amygdalar blood oxygenation leveldependent response to threat-related emotional faces in women with intimate-partner violence posttraumatic stress disorder. Biol Psychiatry 2010; 68: 433-441.

36. Fechir M, Gamer M, Blasius I, et al. Functional imaging of sympathetic activation during mental stress. Neuroimage 2010; 50: 847-854.

37. Baur V, Hänggi J, Rufer M, et al. White matter alterations in social anxiety disorder. J Psychiatr Res. 2011; 45: 1366-1372.

38. Baur V, Hänggi $J$ and Jäncke L. Volumetric associations between uncinate fasciculus, amygdala, and trait anxiety. BMC Neurosci 2012; 13: 4.

39. Baur V, Brühl AB, Herwig U, et al. Evidence of frontotemporal structural hypoconnectivity in social anxiety disorder: A quantitative fiber tractography study. Hum Brain Mapp 2013; 34: 437-446. 